\title{
Measuring Perimeter and Area in Low Resolution Images Using a Fuzzy Approach
}

\author{
Nataša Sladoje $^{1 \star}$, Ingela Nyström ${ }^{1}$, and Punam K. Saha ${ }^{2}$ \\ 1 Centre for Image Analysis, Uppsala, Sweden \\ natasa@cb.uu.se, ingela@cb.uu.se \\ 2 Medical Image Processing Group, Department of Radiology \\ University of Pennsylvania, Philadelphia, PA, USA \\ saha@mipg. upenn. edu
}

\begin{abstract}
To overcome the problems of low quality of image segmentation, as well as significant loss of the data, it seems promising to retain the data inaccuracies as realistic as possible during the image analysis procedures, instead of making hard decisions in the segmentation phase. Such an approach initializes the interest for new image analysis methods, handling grey-level images. Our work on developing shape analysis methods for fuzzy segmented images has resulted in a theoretical foundation for estimators of quantitative properties of digitized objects with fuzzy borders. In this paper, we present results of perimeter, area, and compactness measure estimations obtained by applying a fuzzy approach to digitized objects in low resolution real images.
\end{abstract}

Keywords: Fuzzy shape representation, boundary, accuracy, precision

\section{Introduction}

Fuzzy segmentation methods have been developed in order to reduce the negative effects of the loss of data in the digitization process, caused by crisp (hard) segmentation of a grey-level image, see, e.g., [7]. In that case, instead of working with a binary image, analysis is performed directly on a grey-level image, or on its corresponding fuzzy segmented image.

As we are interested in estimating quantitative properties of fuzzy segmented digital objects, we have developed a perimeter estimation method for fuzzy segmented shapes. Empirical results for synthetic digitized objects, show that a fuzzy segmentation provides more precise measures of area, perimeter, and compactness $\left(P^{2} A\right)$ than a hard segmentation. An increase of the resolution (by itself), provides an improvement of the estimation results [3], both in fuzzy and hard case. However, the increase of the precision of the estimation obtained by taking a fuzzy approach is much more obvious for low resolution images.

\footnotetext{
* The author is supported by a grant from the Swedish Institute.
} 
Our results for small synthetic objects, (i.e., objects digitized at low resolution), are encouraging. We use these results to predict the behaviour of the suggested method applied to real images.

Area, perimeter, and $P^{2} A$ measure estimations obtained for circular objects of known size, from (low resolution) grey-level images, are presented for mathematical and physical phantoms. They confirm reliability of the method. The method is finally applied to estimate the area, perimeter, and compactness measure of a cross-section of the aorta from a magnetic resonance angiography (MRA) image.

\section{Background and Related Works}

In this Section, a brief theoretical background for the estimation methods used in this paper is given. They are derived in [8].

The area and perimeter of a digital fuzzy subset are defined with respect to the definition of area and perimeter of a fuzzy subset in the continuous domain. A fuzzy digital image is a fuzzy step subset, where iso-membership valued sets are determined with respect to the connected sets of pixels having the same grey-level. For the definition of a fuzzy step subset, see [1]. Definitions used:

Definition 1. [10] A fuzzy subset $M$ of a reference set $X \in \mathbf{R}^{n}$ is a set of ordered pairs

$$
M=\left\{\left(x, \mu_{M}(x)\right) \mid x \in X\right\},
$$

where $\mu_{M}: X \rightarrow[0,1]$ is the membership function of $M$ in $X$.

Definition 2. [6] The area $A(M)$ of a fuzzy subset $M$ of a reference set $X$, given by its membership function $\mu_{M}$, is

$$
A(M)=\int_{X} \mu_{M}(x) d x .
$$

Definition 3. [6] The perimeter $P(S)$ of a fuzzy step subset $S$, given by its membership function $\mu_{S}$, is

$$
P(S)=\sum_{\substack{i, j=1 \\ i<j}}^{n+1}\left|s_{i}-s_{j}\right| \cdot l\left(B_{i j}\right),
$$

where $s_{i}=\mu_{S}(x)$ for $x$ from the iso-membership valued set $S_{i}$, and $l\left(B_{i j}\right)$ denotes the length of a border line between two neighbouring iso-membership valued sets of pixels, $S_{i}$ and $S_{j}$.

According to Definition 2, the area of a fuzzy digital object is calculated as the sum of pixel values having their centroid within the object. The main result of [8] is the perimeter estimator derived from Definition 3, by incorporating an efficient estimator of the length of the border line, $l\left(B_{i j}\right)$, between two neighbouring isomembership valued sets of pixels, $S_{i}$ and $S_{j}$, having different grey-levels. For that estimation we use a local approach, relying on measuring elementary moves within the path $l\left(B_{i j}\right)$. 


$$
\begin{array}{|l|l|l|}
\hline z_{1} & z_{2} & z_{3} \\
\hline z_{4} & z_{5} & z_{6} \\
\hline z_{7} & z_{8} & z_{9} \\
\hline
\end{array}
$$

Fig. 1. The $3 \times 3$ neighbourhood of a pixel $z_{5}$ in a $2 D$ digital image.

We assume that, during a segmentation process, most of the image points can be classified as either object or background, but that for some of the points it is hard to make this discrimination. Such points are often located around the border of an object. By determining the extent of their membership to the object/background, the border of an object is defined as a fuzzy subset. We compute the membership value of a pixel as a fraction of its area belonging to the original object. The membership value of the pixels is equal to 1 in the inner — "central" - region of the object, and "radially" decreases toward the border. Pixels in the background have membership value 0 . This allows us to assume that the objects we work with fulfill the property that we call local fuzzy convexity.

Definition 4. (Local fuzzy convexity property) A fuzzy digital object has a local fuzzy convexity property if the $3 \times 3$ neighbourhood of each pixel in the image is a convex fuzzy subset. (For the definition of a convex fuzzy subset see, e.g., [9].)

Let the $3 \times 3$ neighbourhood of a pixel $z_{5}$ be denoted as in Figure 1 . To compute the increase of the membership function, i.e., a measure related to the gradient at $z_{5}$, we calculate

$$
\begin{aligned}
& d_{\text {hor }}=\max \left\{\mu\left(z_{6}\right)-\mu\left(z_{5}\right), \mu\left(z_{4}\right)-\mu\left(z_{5}\right), 0\right\}, \\
& d_{\text {vert }}=\max \left\{\mu\left(z_{2}\right)-\mu\left(z_{5}\right), \mu\left(z_{8}\right)-\mu\left(z_{5}\right), 0\right\},
\end{aligned}
$$

and then assign

$$
d_{\text {max }}=\max \left\{d_{\text {hor }}, d_{\text {vert }}\right\} \quad \text { and } \quad d_{\text {min }}=\min \left\{d_{\text {hor }}, d_{\text {vert }}\right\} .
$$

The contribution $\operatorname{per}\left(z_{5}\right)$ of the observed pixel $z_{5}$ to the estimated perimeter is

$$
\operatorname{per}\left(z_{5}\right)=d_{\text {min }} \cdot b \text {-step }+\left(d_{\text {max }}-d_{\text {min }}\right) \cdot a \text {-step, }
$$

where $a$-step and $b$-step are the estimates (weights) of the isothetic and the diagonal distance between two neighbouring pixels, respectively, see [2]. We use $a_{n \rightarrow \infty} M S E \approx 0.948$ and $b_{n \rightarrow \infty} M S E \approx 1.343$ for the $a$ - and $b$-step weights [4] to minimize the expected mean square error $(M S E)$ for measurements of the lengths of long line segments $(n \rightarrow \infty)$.

For small objects, the subtraction of an appropriately chosen constant value from the estimated perimeter compensates for the over-estimation caused by digitization. We have determined the correction factor $c_{c o r r}$ as the difference between the mean estimated perimeter value of 10,000 (hard) disks of radius 
1 pixel, randomly positioned in a grid, and the real perimeter value of a disk of the same size, which gives $c_{\text {corr }}=0.689078$.

We compute the perimeter of a fuzzy segmented digitized object $S$ by using the following equation

$$
P(S)=\sum_{z \in I} \operatorname{per}(z)-c_{c o r r},
$$

where $\operatorname{per}(z)$ is the contribution to the perimeter of any pixel $z$ in the image $I$.

\section{Results for Fuzzy Segmented Images}

The purpose of this paper is to report on area and perimeter estimations when the methods suggested in [8] and described above are applied to fuzzy segmented images. The $P^{2} A$ compactness measure, determined as

$$
P^{2} A(S)=\frac{\operatorname{perimeter}^{2}(S)}{4 \pi \cdot \operatorname{area}(S)},
$$

is also estimated. Our intention is to show that our perimeter and area estimates provide high resemblance of binary and continuous objects, regarding isoperimetric inequality, (perimeter $(S))^{2} \geq 4 \pi \cdot \operatorname{area}(S)$.

\subsection{Mathematical phantoms}

Tests are done for 6, 800 randomly positioned disks (the centres are uniformly distributed over the unit pixel area), 100 for each of 68 real-valued radii up to 20 pixels, while different sub-sample factors $(1,4,8$, and 16), corresponding to different levels of fuzziness, are used. The sub-sample factor 1 corresponds to hard segmentation. For each size and sub-sample factor, we determine the mean of the estimated values, as well as maximum and minimum estimates. The results are presented in Figure 2. For more details, see [8], where the estimation results for other shapes are presented as well. Here, we will give the conclusions.

The common property of estimates based on a hard segmentation is their rather low precision for small objects. By introducing fuzziness in the segmentation procedure, much better results, in terms of precision, are obtained. For disks of radius 5 pixels, the maximal error for the area estimation is reduced from $4 \%$ (in the hard case) to $0.3 \%$ (if fuzzy segmentation with sub-sample factor 8 is used), while the maximal error for the perimeter estimation (Eq. 1) of such disks is reduced from $6 \%$ (hard) to $3 \%$ (fuzzy). For the $P^{2} A$ measure estimation, the values obtained for fuzzy segmented objects are not only precise, but never less than 1 , which is in accordance with the well-known property of the continuous circles, but does not hold for hard segmented digitized objects. 

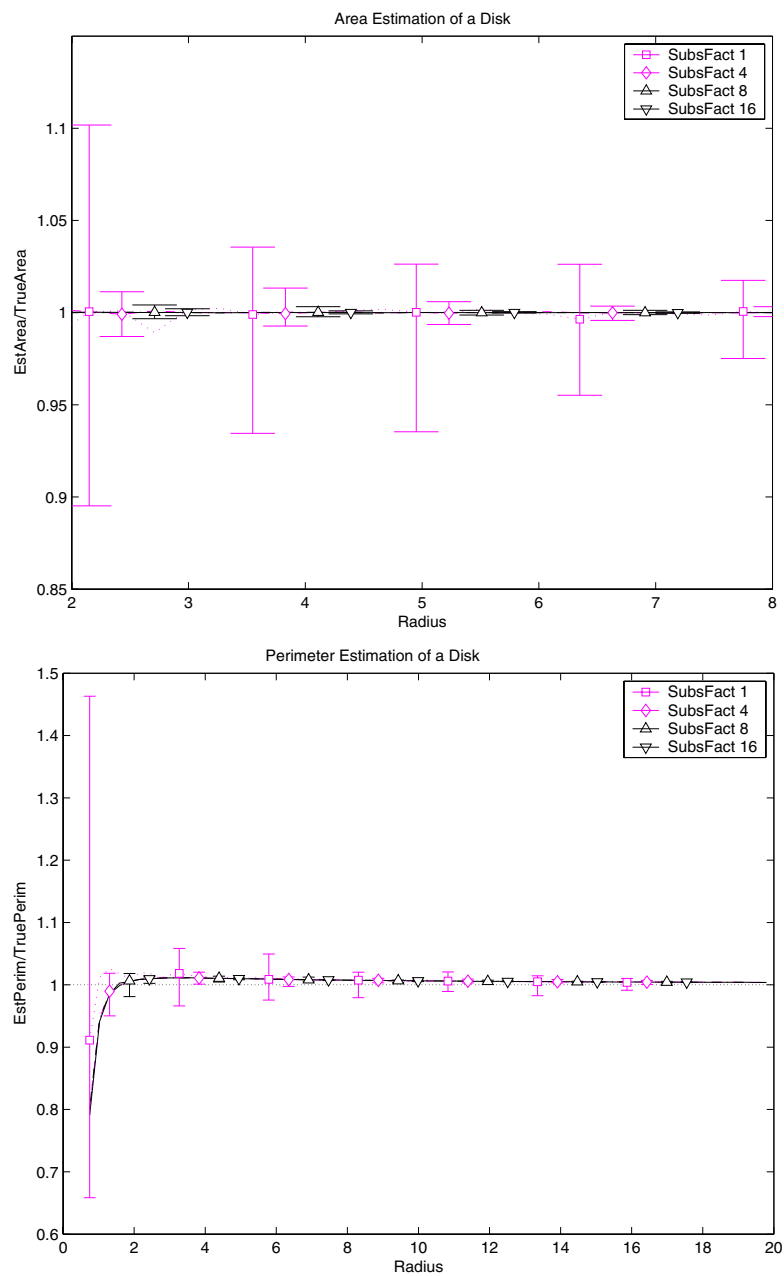

Fig. 2. Measurements of disks randomly positioned in a grid. Top: Area estimation results for very small disks. Bottom: Perimeter estimation results for small disks.

\subsection{Physical phantoms}

We acquired images of holes made by a perforator in a piece of blue paper by using an ordinary scanner set at resolutions 75-200 dpi. The true diameter of the holes is $5.9 \mathrm{~mm}$. By choosing the red band of the original colour image, we get a light circular object on a dark background, while the border of the object is not strictly defined as dark or light, but fuzzy segmented, see Figure 3(a, b).

Only a few processing steps are needed to obtain objects that fulfill the conditions for the perimeter estimation method [8]. By grey-level thresholding, we define the inner region, containing pixels set to the maximal value (i.e., having 


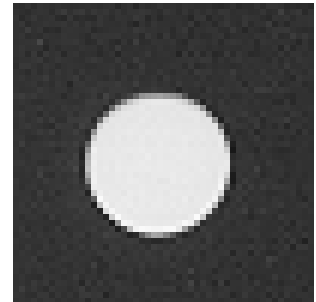

(a)

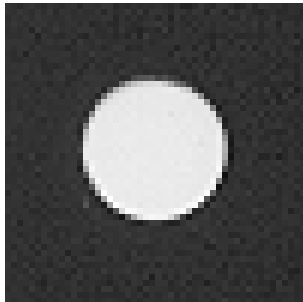

(b)

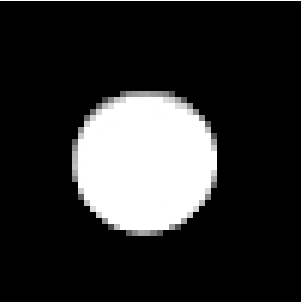

(c)

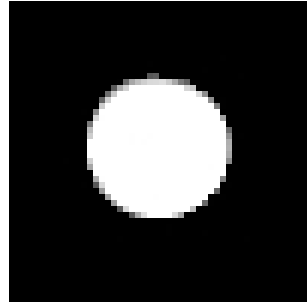

(d)

Fig. 3. (a), (b) Circular objects extracted from the original image (resolution 100 dpi). (c), (d) Fuzzy segmented objects obtained from (a) and (b).

membership value 1), and background region, containing pixels set to 0 as their membership to the object. The border points are set to values proportional to their grey-level. Local convexity is checked for. Example images are presented in Figure $3(\mathrm{c}, \mathrm{d})$. The perimeter, area, and $P^{2} A$ measure estimations, presented in Table 1 , are very encouraging.

Table 1. Perimeter $(\mathrm{mm})$, area $\left(\mathrm{mm}^{2}\right)$, and $P^{2} A$ estimations of circular objects with fuzzy border, obtained from the images scanned at different resolutions (75-200 dpi); percentual errors (Per. Error and Area Error) are also given.

\begin{tabular}{|c|c||c|c||c|c||c|}
\hline object $_{\mathbf{d p i}}$ & Radius & Perimeter & Per. Error & Area & Area Error & $\mathbf{P}^{\mathbf{2}} \mathbf{A}$ \\
\hline \hline real object & $2.95 \mathrm{~mm}$ & 18.54 & - & 27.34 & - & 1.00 \\
\hline \hline object $1_{75}$ & $\approx 8$ pixels & 18.89 & 1.92 & 27.91 & 2.06 & 1.02 \\
\hline object $2_{75}$ & $\approx 8$ pixels & 18.81 & 1.51 & 27.91 & 2.09 & 1.01 \\
\hline \hline object $1_{100}$ & $\approx 11$ pixels & 18.89 & 1.92 & 28.23 & 3.27 & 1.01 \\
\hline object $2_{100}$ & $\approx 11$ pixels & 18.97 & 2.32 & 28.05 & 2.60 & 1.02 \\
\hline \hline object $1_{150}$ & $\approx 17$ pixels & 18.83 & 1.59 & 27.96 & 2.27 & 1.01 \\
\hline object $2_{150}$ & $\approx 17$ pixels & 18.85 & 1.70 & 27.88 & 1.94 & 1.02 \\
\hline \hline object $1_{200}$ & $\approx 22$ pixels & 18.74 & 1.10 & 27.53 & 0.68 & 1.02 \\
\hline object $2_{200}$ & $\approx 22$ pixels & 18.83 & 1.59 & 27.51 & 0.61 & 1.03 \\
\hline
\end{tabular}

We conclude that all estimations are rather accurate and in reasonably good accordance with the theoretical results. In this case, where the fuzziness is defined in proportion to the grey-levels of the digitized real image, the percentual errors are just slightly larger than for the synthetic images of the disks where the fuzzy border is defined by using area coverage. 


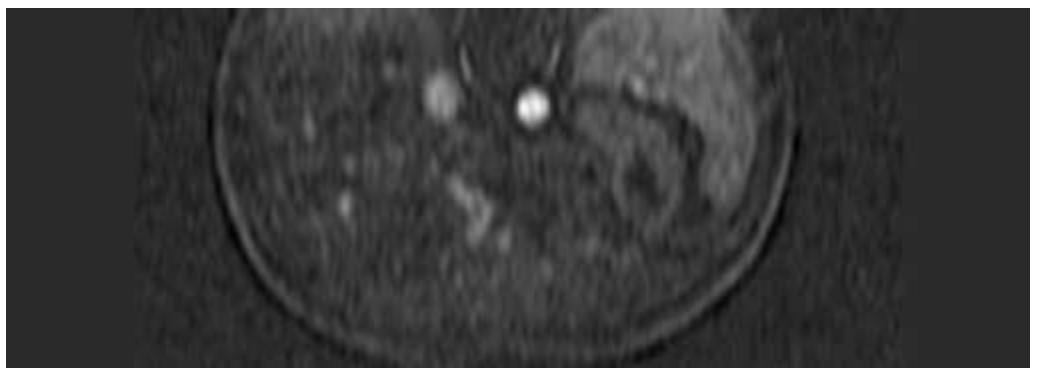

(a)

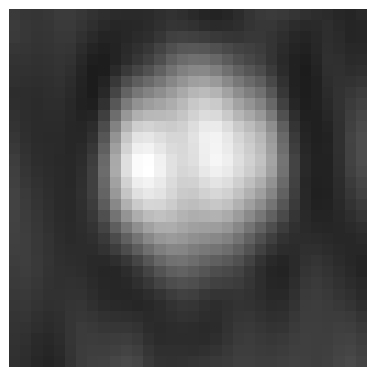

(b)

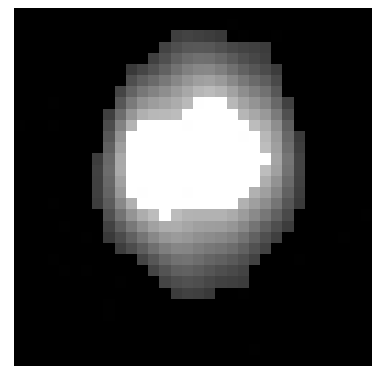

(c)

Fig. 4. (a) $512 \times 180$ pixel cross-section of a (3D) MRA image. (b) Extracted subimage containing aorta, the bright circular object. (c) Fuzzy segmented aorta.

\subsection{Real objects}

Finally, we applied the proposed estimation methods to a real object from a magnetic resonance angiography (MRA) image of the human abdomen, a $512 \times 180$ cross-section of the aorta with pixel size $0.78125 \mathrm{~mm}$, see Figure 4(a). After some pre-processing steps, including grey-scale morphological operations, and thresholding to define inner and outer region, we obtained a locally convex circular object with a fuzzy border, see Figure 4(b). In this case, the border is rather thick, since making a hard decision in defining the object is difficult.

The estimation results are EstPerimeter $=40.49 \mathrm{~mm}$, EstArea $=128.51$ $\mathrm{mm}^{2}$, and $E s t P^{2} A=1.06$. This corresponds to approximately $12.8 \mathrm{~mm}$ in diameter, which is quite reasonable for the aorta at this level [5] (from an 11-year old child).

Our main goal is to show that our perimeter estimation method (and also area and compactness estimation methods) can be applied to such "vaguely" defined objects. Here, we have used a fuzzy membership function which can be easily and naturally derived from grey-level images obtained by many imaging techniques; the membership values are proportional to the area coverage, which we also used in our theoretical study. 


\section{Conclusions}

Defining (the border of) a digitized object as a fuzzy subset seems to be very promising, considering estimates of quantitative properties of the original object. Results of the estimation of area, perimeter, and $P^{2} A$ (compactness) measure, for synthetic locally convex objects are generally improved when the membership of a digital point to a set is allowed to be not just 0 or 1 , but also values between these two extrema. This approach significantly improves the estimations, as compared to the hard segmentation, in the case of low resolution images.

Application of the proposed estimation methods to real images shows that the approach of retaining fuzziness in the object border provides a simple and natural segmentation method when hard decisions about the object definition would be difficult to make. The MRA image used in this paper, and medical images obtained by other devices, contain objects that can be defined as locally convex objects with fuzzy borders. The high precision and accuracy of the presented estimation results make us confident that the theoretical results derived in [8] can be efficiently applied to a wide range of real images.

\section{Acknowledgements}

The following colleagues at Centre for Image Analysis, Uppsala, Sweden, are acknowledged: Prof. Gunilla Borgefors and Dr. Joakim Lindblad for their scientific support, and Xavier Tizon for providing the MRA image.

\section{References}

1. A. Bogomolny. On the perimeter and area of fuzzy sets. Fuzzy Sets 6 Systems, 23:257-269, 1987

2. G. Borgefors. Distance transformations in digital images. Computer Vision, Graphics, and Image Processing, 34:344-371, 1986

3. D. Coeurjolly and R. Klette. A comparative evaluation of of length estimators. Proc. of Intern. Conf. on Pattern Recognition (ICPR 2002), IV:330-334, 2002

4. L. Dorst and A. W. M. Smeulders. Length estimators for digitized contours. Computer Vision, Graphics, and Image Processing, 40:311-333, 1987

5. M. Huonker, et al. Size and blood flow of central and peripheral arteries in highly trained able-bodied and disabled athletes. Journal of Applied Physiology, 2002

6. A. Rosenfeld and S. Haber. The perimeter of a fuzzy subset. Pattern Recognition, 18:125-130, 1985

7. P. K. Saha and J. K. Udupa. Relative fuzzy connectedness among multiple objects: Theory, algorithms, and applications in image segmentation. Computer Vision and Image Understanding, 82(1):42-56, 2001

8. N. Sladoje, I. Nyström, and P. K. Saha. Perimeter and area estimations of digitized objects with fuzzy border. Submitted.

9. X. Yang. Some properties of convex fuzzy sets. Fuzzy Sets ES Systems, 72:129-132, 1995

10. L. Zadeh. Fuzzy sets. Information and Control, 8:338-353, 1965 